\title{
Localised cutaneous microvascular adaptation to exercise training
}

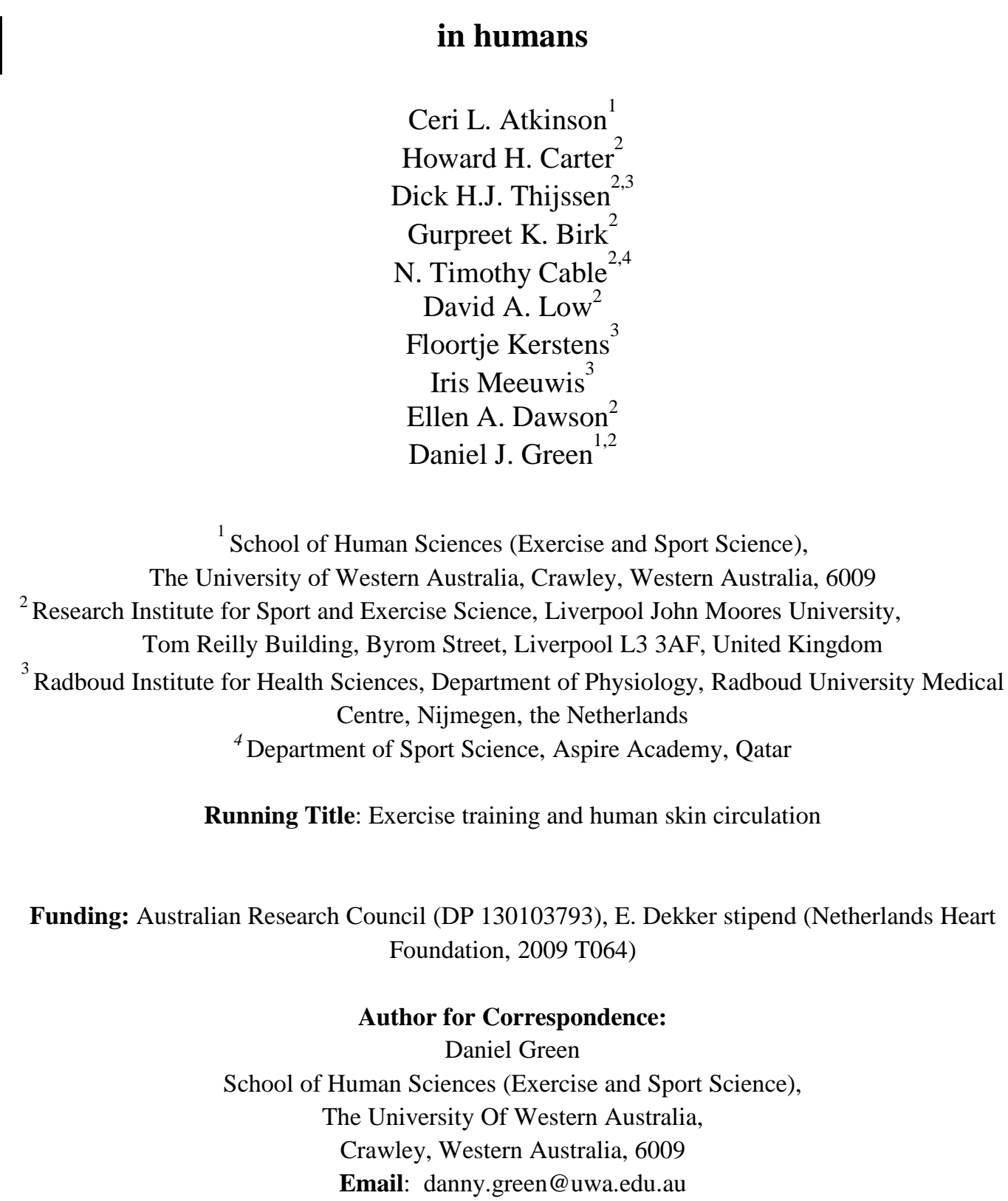

Funding: Australian Research Council (DP 130103793), E. Dekker stipend (Netherlands Heart Foundation, 2009 T064)

Author for Correspondence:

Daniel Green

School of Human Sciences (Exercise and Sport Science),

The University Of Western Australia, Crawley, Western Australia, 6009

Email: danny.green@uwa.edu.au 


\begin{abstract}
Purpose. Exercise training induces adaptation in conduit and resistance arteries in humans, partly as a consequence of repeated elevation in blood flow and shear stress. The stimuli associated with intrinsic cutaneous microvascular adaptation to exercise training have been less comprehensively studied.

Methods. We studied 14 subjects who completed 8-weeks cycle ergometer training, with partial cuff inflation on one forearm to unilaterally attenuate cutaneous blood flow responses during each exercise-training bout. Before and after training, bilateral forearm skin microvascular dilation was determined using cutaneous vascular conductance (CVC: skin flux/blood pressure) responses to gradual localised heater disk stimulation performed at rest (33, 40, 42 and $44^{\circ} \mathrm{C}$ ). Results. Cycle exercise induced significant increases in forearm cutaneous flux and temperature, which were attenuated in the cuffed arm (2-way ANOVA interaction-effect; $P<0.01$ ). We found that forearm CVC at $42^{\circ} \mathrm{C}$ and $44^{\circ} \mathrm{C}$ was significantly lower in the uncuffed arm following 8-weeks of cycle training $(P<0.01)$, whereas no changes were apparent in the contralateral cuffed arm $(P=0.77$, interaction-effect $P=0.01)$.
\end{abstract}

Conclusions. Lower limb exercise training in healthy young men leads to lower CVC-responses to a local heating stimulus, an adaptation mediated, at least partly, by a mechanism related to episodic increases in skin blood flow and/or skin temperature.

Keywords: skin microcirculation; blood flow; exercise training 


\author{
58 Abbreviations \\ 59 BMI - body mass index \\ 60 CVC - cutaneous vascular conductance \\ 61 HRMax- Heart rate max \\ 62 PU - Perfusion units \\ 63 SNP - sodium nitroprusside
}




\section{Introduction}

Exercise training has strong and independent cardioprotective effects $\{$ Blair, $2009 \# 2719$ \} that may be partly mediated through the direct impact of exercise on the vasculature \{Green, 2008 \#2059;Joyner, 2009 \#2509;Green, 2017 \#3215\}. In conduit and resistance arteries, dynamic exercise training using large muscle groups leads to generalised effects on the vasculature \{Thijssen, 2012 \#3199;Green, 2011 \#2708;Silber, 1991 \#167;Snell, 1987 \#171\}. For example, cycle ergometer exercise training, which specifically avoided hand-gripping or use of the upper limb musculature, induced brachial artery adaptation \{Birk, 2012 \#2970;Tinken, 2008 \#2979\}. Interestingly, when exercise-mediated increases in brachial artery blood flow were attenuated during exercise bouts (via unilateral partial cuff inflation), these adaptations were no longer apparent, implying a role for haemodynamic forces, such as shear stress, as important stimuli to conduit artery adaptation in response to exercise training in humans \{Birk, 2012 \#2970; Tinken, $2010 \# 2491\}$.

Whilst conduit and larger resistance arteries adapt to exercise training by enhancing function and then remodelling to increase their diameter \{Green, 2017 \#3215;Tinken, 2008 \#2979;Laughlin, $1995 \# 766$ \}, studies of microvascular adaptation in skeletal muscle have proposed that increases in capillarity and microvaseular volume-in response to training may counteract reductions in red cell transit time, enabling increased oxygen extraction \{Krustrup, $2004 \# 3005$ \}. In this context, it is well established that the mode of adaptation to training differs according to rank order along the arterial tree; larger arteries primarily respond to shear stress and other hemodynamic stimuli via cross sectional enlargement \{Rowley, 2011 \#2829;Newcomer, 2011 \#2723\}, whereas microvessels respond to these stimuli (and hypoxia) by budding and sprouting in grow and 
number, with the magnitude of change dependent upon the duration and intensity of the exercise intervention $\{$ Cocks, 2013 \#3220;Andersen, 1977 \#3221;Brown, 2003 \#1635\}.

The role of repeated exercise and shear stress in the adaptation of cutaneous microvessels is less well understood, despite the obvious relevance of changes in the structure and function of these vessels to microvascular disease \{Simmons, 2011 \#2963;Holowatz, 2008 \#2501\}. Some evidence suggests that exercise training induces intrinsic adaptation in the cutaneous microvasculature Black, 2008 \#2096;Simmons, 2011 \#2963\}, whilst we recently completed a series of studies, involving repeated localised and systemic heating, which suggested that cutaneous microvascular adaptation may depend upon local skin temperature changes in addition to changes in blood flow and shear stress that occur as a consequence of elevation in core temperature \{Green, 2010 \#2601;Carter, 2014 \#3070\}. The contribution of these important physiological stimuli to cutaneous adaptation in response to leg exercise training is currently unknown.

The primary aim of this study was to examine the role played by repeated increases in blood flow in localised adaptations in the skin microcirculation of the upper limbs, following 8-weeks of cycling exercise. To address this aim, we partially inflated a pneumatic cuff around one forearm during each exercise bout, to unilaterally attenuate exercise-induced hyperaemia \{Tinken, 2009 \#2488;Tinken, 2010 \#2491\}. Before and after the intervention, skin blood flow responses were assessed at rest using a local heating protocol. We chose to use a localised disk heating protocol as a tool to assess intrinsic microvascular function-adaptation in the skin because it is well established that this approach induces a largely nitric oxide-mediated 
vasodilation response, providing provides an intrinsic microvascular index that is independent of reflex neurovascular control \{Minson, 2001 \#2965;Black, 2008 \#2096\}. We hypothesised that cycle exercise training, by inducing repeated episodic increases in cutaneous blood flow would increase forearm skin vasodilator capacity in response to a localised heating stimulus.

\section{Materials and Methods}

\section{Ethical Approval}

All study procedures complied with the Declaration of Helsinki and were approved by the Human Research Ethics Committee at Liverpool John Moores University and The University of Western Australia. All subjects provided written, informed consent before participating in the study.

\section{Subjects}

Fourteen healthy men (Age: $25 \pm 2$ years, Height: $180 \pm 5 \mathrm{~cm}$, Weight: $76 \pm 13 \mathrm{~kg}$ and BMI: $23 \pm 3$ kg. $\mathrm{m}^{-2}$ ) were recruited to participate in an exercise training experiment. A further 6 male subjects

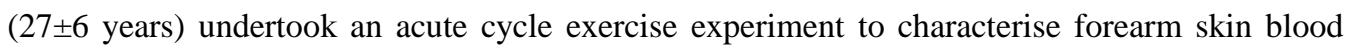
flow changes. No subject had cardiovascular disease, diabetes, insulin resistance or possessed cardiovascular risk factors such as hypercholestolaemia or hypertension. Subjects who smoked or were on medication of any type were excluded. Participants at pre-study entry were either classified as sedentary or recreationally active ( $<5$ hours per week). All sedentary participants only took part in the prescribed 3 x 30 min sessions per week across the 8 weeks and no new activity was introduced outside of this. All recreationally active participants were asked to keep 
their activity levels consistent throughout the 8 week intervention so that the additional $3 \times 30$ mins a week were in addition to pre-entry exercise levels.

\section{Acute experiment $(n=6)$ : Effect of leg cycle exercise on skin microvascular responses \pm forearm cuff inflation}

Participants undertook 30 mins of cycle exercise (Monark 874E, Sweden) at 80\% HRmax (agepredicted). Throughout the exercise bout a pneumatic cuff (6cm in width: SC5D Hokanson cuff, Bellevue USA) was placed around one forearm immediately below the cubital crease and inflated to 60mmHg. Previous studies have demonstrated that placement and inflation of a forearm cuff in this manner attenuates brachial artery blood flow \{Birk, 2012 \#2970;Thijssen, 2009 \#2482;Tinken, 2010 \#2491;Tinken, 2009 \#2488\}. The contralateral arm remained uncuffed during the exercise bout. Subjects rested for 10-mins on the ergometer in a quiet, temperature controlled room. Baseline bilateral forearm skin blood flows using laser-Doppler flowmetry (Model 413, Periflux 5001 System, Perimed AB) were then recorded for 2.5 mins, followed by unilateral cuff inflation to $60 \mathrm{mmHg}$ for 2.5 mins. Skin blood flow data were recorded in both arms on the ventral side of the forearm during the exercise bout and averaged in 2.5-min bins along with skin temperature using thermistors (Squirrel 1000 series, Grant Instruments, Cambridge, United Kingdom). Placement of skin thermistors and laser Doppler probes was the same on both arms and always distal to the cuff site. In order to avoid any instances of handgripping, participants were instructed to rest both arms on the handle bars of either side of the bike. 


\section{Chronic experiment (n=14): Effect of leg cycle exercise on skin microvascular responses \pm} forearm cuff inflation

Exercise training was performed over an 8-week period with subjects visiting the laboratory 3 times per week. Each of these 24 sessions was supervised and consisted of 30 mins of cycle exercise (80\% age-predicted HRmax, cadence $60 \mathrm{rpm})$, performed at the same time of day. As above, a pneumatic blood pressure cuff was placed below the cubital crease on one forearm of each subject and inflated to $60 \mathrm{mmHg}$ throughout each $30 \mathrm{~min}$ exercise training bout. The arm selected for cuff placement was randomised, but once selected, it remained consistent across the 8-week training period for any given participant.

\section{Gradual local heating protocol}

Assessments were performed before and after the 8-week cycling exercise training program at rest. All studies were conducted in a quiet, temperature controlled environment and each visit for a given subject was performed at the same time of day. Subjects were asked to fast for 8 hours, abstain from alcohol and caffeine for 16 hours, and not to perform any exercise for 24 hours. Probe placement sites were selected on each forearm and the location of these sites was recorded using photographs and distances from anatomical landmarks, so that repeated measures were collected at the same skin site. In a previous study, we examined the day-day reproducibility of forearm skin responses to our local heating protocol. Coefficients of variation of 17.1 and $14.4 \%$ were obtained when skin responses were presented as absolute cutaneous vascular conductance (CVC; at 42 and $44^{\circ} \mathrm{C}$, respectively) \{Dawson, $\left.2015 \# 3122\right\}$. 
Upon arrival, subjects were seated and instrumented for $\sim 20$ mins before beginning the $\sim 90$ min heating protocol. Local heater disks (Perimed 455, Stockholm, Sweden) were placed on the forearms using double-sided adhesive rings. The laser Doppler probes, each with a 7-laser array (Model 413, Periflux 5001 System, Perimed AB), were then fitted into the middle of these localised heating disks to record change in red blood cell flux (in perfusion units, PU) \{Cracowski, 2006 \#2093\}. Once instrumented, recording commenced and the heater disk temperatures were increased to $33^{\circ} \mathrm{C}$ and maintained at this temperature for a 10 min baseline period. Upon completion of this baseline period, increments in heater disk temperature were gradual, so as to minimise the influence of the axon reflex on the skin blood flow response \{Minson, $2001 \# 2965$ \}. Heat increased in controlled increments of $0.5^{\circ} \mathrm{C}$ every 2.5 mins until $42^{\circ} \mathrm{C}$ was reached. Once the probes reached $42^{\circ} \mathrm{C}$, they remained at this temperature for a period of 30 mins. Finally, heater disk temperatures were increased to $44^{\circ} \mathrm{C}$ for another 20 mins to induce a maximal skin blood flow response. Previous studies have suggested that heating to the levels used in the present study results in similar CVC increases to those observed in response to infusion of SNP \{Kellogg, 1999 \#2108;Cracowski, 2011 \#3200\}, indicating that maximal responses are indeed obtained and that these reflect the structural capacity of the microvasculature. Resting blood pressure was recorded every 15 min using an automated sphygmomanometer placed around the ankle (Dinamap; GE Pro 300V2, Tampa, FL). Blood pressure measures taken at the ankle were later corrected for the hydrostatic column by multiplying the vertical height difference between the right atrium and the ankle $(\mathrm{cm})$ by 0.766 to convert to mmHg \{Groothuis, 2008 \#2959\}, this value was then subtracted from the raw blood pressure value acquired at the ankle. Converted blood pressure values were then used to calculate CVC, which accounts for skin blood flow changes occurring as a result of changes in 
blood pressure \{Cracowski, 2006 \#2093\}. All laser Doppler measurements were relayed and graphed in real time via a Powerlab onto a laptop running LabChart 7 (AD Instruments, Sydney, Australia).

\section{Data analysis and statistics}

Skin blood flow (PU) from the cuffed and uncuffed arms was averaged across 1-minute intervals. Calibration of the laser Doppler probes was undertaken before and after the experiments using two generic points, 0 and $250 \mathrm{PU}$, in accordance with calibration guidelines using a zeroing disk and motility standard (Periflux System, Perimed AB). We measured and analysed the $2.5 \mathrm{~min}$ increment at $40^{\circ} \mathrm{C}$, as well 5-min averages of data from the plateau-phases at $42^{\circ} \mathrm{C}$ and $44^{\circ} \mathrm{C}$. Measurements in PU were converted to CVC which was calculated as PU/Dinamap mean arterial pressure (MAP).

Statistical analyses were performed using SPSS 21.0 (SPSS, Chicago, Illinois) software. All data are reported as mean (SD) unless stated otherwise, while statistical significance was assumed at $\mathrm{P} \leq 0.05$. Initially, a linear mixed model was conducted (with time and cuff inflation as factors) for both skin temperature and skin blood flow during the acute study. A second linear mixed model was used with local heating (33, 40, 42 and $44^{\circ} \mathrm{C}$ ) and time (Week $0 \& 8$ ) as factors in order to assess change in CVC responses to the 8-week intervention in the uncuffed and cuffed arm. Finally, a linear mixed model was performed on delta change CVC scores (Week 8 minus Week 0) with cuff and heating as factors. Post-hoc analysis was performed using the least significant difference (LSD) method for pair-wise multiple comparisons when a significant main effect was observed \{Perneger, 1998 \#3201\}. 


\section{Results}

\section{Acute experiment $(n=6)$ : Effect of leg cycle exercise on skin microvascular responses \pm forearm cuff inflation}

Results of a linear mixed model revealed that heart rate increased significantly during the leg cycling exercise bout (Figure 1A). The increase in HR observed prior to exercise from the -2.5 to 0 min time points may bewe attribute to feed-forward-an anticipatory reflexesresponse. Skin temperature in the uncuffed forearm increased modestly, but significantly, during exercise (by Comment [DG3]: Add a ref about HR anticipatory feed forward response

$1.8^{\circ} \mathrm{C}$, Figure $\left.1 \mathrm{~B}\right)$. However, no change in skin temperature was observed during leg cycling exercise in the cuffed arm (Figure 1B). No significant differences were evident in skin blood flow between the cuffed and uncuffed arms in the baseline period before the cuff was inflated (13.1 \pm 3.5 vs. $15.5 \pm 9.1 \mathrm{PU}$, respectively, $P=0.41)$. After the onset of leg cycling exercise, there was a significant increase in skin blood flow in the uncuffed arm, whilst the increase in flow was significantly attenuated in the cuffed arm (linear mixed model; interaction effect between exercise and cuff inflation of $P<0.01$, Figure 1C).

\section{Chronic experiment (n=14): Effect of leg cycle exercise on skin microvascular responses \pm}

\section{forearm cuff inflation}

Across the 8-week exercise training period, there was $97 \%$ adherence to the training sessions in the 14 subjects. The efficacy of our training intervention is indicated by a decrease in the average resting heart rate after 8 weeks of cycle exercise training $(67 \pm 11 \mathrm{bpm}$ vs. $58 \pm 9$; t-test: $P=0.01$ ). In addition, to maintain exercise $\mathrm{HR}$ at $80 \% \mathrm{HRmax}$, the workload performed in the 
exercise sessions increased significantly over the 8 weeks of training, from $129 \pm 19 \mathrm{~W}$ to $154 \pm 21 \mathrm{~W}$ at week $8(P<0.01)$. Resting MAP did not change after training $(89 \pm 8 \mathrm{vs} 88 \pm 6 \mathrm{mmHg}$, $\mathrm{P}=0.57)$.

\section{Gradual local heating protocol}

Figure 2 shows a representative response to local heating in one subject throughout the temperature increments while Figure 3 presents the responses to our local heating protocol before and after training in the cuffed and uncuffed arms. This fFigure 3 presents CVC data in absolute values, rather than normalised to the peak $\left(44^{\circ} \mathrm{C}\right)$ maximum. This is because we wished to understand whether the peak responses per se were elevated, possibly indicative of changes in microvascular capacity. Values for $\mathrm{CVC}$ at baseline $\left(33^{\circ} \mathrm{C}\right)$, heating $\left(40^{\circ} \mathrm{C}\right)$ and during the plateau at $42^{\circ} \mathrm{C}$ and $44^{\circ} \mathrm{C}$, in the cuffed and uncuffed limbs were similar prior to training (Pvalues: $0.32,0.74,0.51$ and 0.76 , respectively). A linear mixed model revealed a significantly lower CVC following training at $42^{\circ} \mathrm{C}$ and $44^{\circ} \mathrm{C}$ (Figure $3 \mathrm{~A}$ ) in the uncuffed arm (interaction effect $P=0.01$, time effect $42^{\circ} \mathrm{C} P \leq 0.01$, time effect $44^{\circ} \mathrm{C} P \leq 0.01$ ). However, in the contra-lateral cuffed arm, CVC did not change as a result of training (Figure 3B) (interaction effect $P=0.69$ ). A linear mixed model for changes in CVC (expressed as CVC Week 8 minus CVC Week 0) between uncuffed vs. cuffed revealed a significant effect for cuff inflation $(P<0.01)$.

\section{Discussion}

The aims of the present study were to describe the impact of cycle exercise training on maximal forearm skin blood flow responses to local heating and to determine whether adaptations were 
dependent upon repeated exposure to increases in blood flow. Our experimental manipulation, involving partial cuff inflation on one arm, was successful in unilaterally manipulating forearm skin blood flow and temperature during leg exercise. Our principal finding, in contrast to our hypothesis, is that cycle exercise training significantly decreased forearm CVC-responses to local heating in the uncuffed forearm. Our findings suggest that this adaptation was mediated by repeated increases in skin blood flow and/or temperature, as no changes were observed in the cuffed forearm which was exposed to significantly lower levels of blood flow/temperature during each cycle exercise training session (Figure 1).

Elicitation of skin blood flow responses via local heating is commonly used for the-assessment of the cutaneous vasculaturer function, as it produces a largely nitric oxide-mediated response \{Boignard, 2005 \#3216;Minson, 2002 \#2092;Smith, 2011 \#3217;Colberg, 2005 \#3218\}. However, few Fewstudies have focussed on the mechanism/s that-modificationy of the localised cutaneous microvasculaturef function-following exercise training in humans \{Boegli, 2003 \#2126;Black, 2008 \#2096\}. Previous studies by our group have utilised exercise-independent interventions to determine the role of repeated increases in skin blood flow on cutaneous vascular function, and found that episodic increases in flow are obligatory for enhanced responsiveness to the local heating test above \{Green, $2010 \# 2601$;Carter, $2014 \# 3070$ \}. It is also well documented that repeated increases in shear stress is-act as a potent stimulus for improved endothelial vascular adaptation and NO availability in larger conduit and resistance arteries in humans \{Hambrecht, 2003 \#1543;Tinken, 2010 \#2491\}. These findings informed the rationale for the present study, in which exercise training was used as a stimulus, but the changes in forearm blood flow and shear stress associated with cycle exercise were clamped unilaterally. 
No previous study has attempted to investigate the role of repeated increases in blood flow on the intrinsic function of the-skin vessels following an exercise intervention. We hypothesised that cycle exercise training would increase skin blood flow responsiveness to local heating, an adaptation mediated by repeated increases in skin blood flow and/or temperature, consistent with the studies above \{Green, 2010 \#2601;Carter, 2014 \#3070\}. _On first pass, our finding in the current study of lower peak CVC responses following the intervention exercise training-suggests that exercise training may have decreased the release of vasodilator stimuli in response to local heating such as nitric oxide. However, the skin circulation is highly complex, and is governed by a number of local and systemic mechanisms \{Charkoudian, $2003 \# 3131\}_{2}$. . The - and the possibility that exercise training induces decreased microvascular function seems unlikely. Moreover, the responses to sustained skin heating to $44^{\circ} \mathrm{C}$ provide an index of peak microvascular vasodilation, commonly used as an index of structural, rather than functional, vascular adaptation in humans (). It is relevant that, in a recent study, we observed a similar decrease in CVC responses to local heater stimulation following an intervention in which we prevented increases in skin temperature during repeated increases in skin blood flow fGarter, $2014 \# 3070\}$. This raises the possibility that interactions between blood flow and the increase in temperature that such flow delivers to the skin may have interactive effects in terms of adaptation in skin microvessels. In this context, it is established that heat shock proteins (especially HSP90) and nitric oxide interact and amplify vasodilator responses \{Garcia-Gardeña, 1998 \#2966; Shastry, 2002 \#2967\}. It is also important to note that, despite the decreased heater disk skin blood flow responsiveness observed in our previous lower limb heating study, a beneficial adaptation was observed following the intervention when skin blood flow was plotted 
against core temperature during passive heating \{Carter, 2014 \#3070\}. We-Of interest, also note

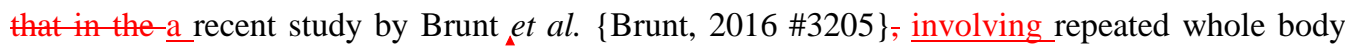
heating for 8 weeks induced improvements in local skin vascular function, but non-significant decreases in maximal local heating responses (5.5\% decrease in CVCmax), consistent with our Formatted: Font: Italic data. Collectively, these findings argue against the notion that the our findings of the current study-infer detrimental functional adaptations in the skin microvessels and that, rather, they may reflect changes in microvascular structure.

\footnotetext{
Given the unexpected nature of our findings we propose an alternate, albeit speculative, explanation relating to the possibility that exercise training induces structural adaptations in the skin microcirculation. This explanation is based on the supported by established findings in both resistance and conduit arteries, that exercise training induces arterial remodelling and structural arterial enlargement \{Haskell, 1993 \#2626;Naylor, 2005 \#2842;Green, 1994 \#2850;Haskell, 1993 \#2626;Rowley, 2011 \#2829;Tinken, 2008 \#2979\}. Such, findings are supported by extensive animal literature \{Langille, $1986 \# 275$ \}. In the current study, the decrease in absolute values for CVC that we observed in the uncuffed arm is not likely to be the result of a decrease in total blood flow to the forearm, since resting brachial artery blood flow [published in \{Birk, 2012 \#2970\}] remained unchanged did not decrease after 8 weeks cycle exercise training (43.3 \pm 20.2 vs $57.0 \pm 28.5 \mathrm{~mL} / \mathrm{min}, \mathrm{P}=0.14)$. We therefore speculate that the decrease in skin flux that we observed following training in response to local skin heating may reflect enlargement of microvessels and/or an increase in the number of capillaries, in that larger microvascular beds should be associated with prolonged red cell transit time. Whilst we admit that such structural adaptation is a novel and speculative proposal in terms of the human cutaneous vasculature,
} 
some supportive evidence for microvascular adaptation after exercise training is provided by studies of skeletal muscle capillary density \{Fernandes, 2012 \#3202;Fernandes, 2012 \#3203\}. Indeed, it has been suggested that increased microcirculatory volume in skeletal muscle following exercise training would counteract higher muscle blood flows, allowing sufficient time for extraction of oxygen \{Krustrup, $2004 \# 3005$ \}. In support of this notion, Glieman et al. observed a training-induced increase in skeletal muscle capillary to fibre ratio as well as increases in capillary lumen area \{Gliemann, 2015 \#3204\}. These interesting findings in skeletal muscle suggest that whilst larger (conduit and feed) arteries adapt both functionally and structurally to increase flow \{Green, $2017 \# 3215$ \}, there may be simultaneous changes in the microvessels that favour prolongation in red blood cell transit time to optimise gas exchange. This is consistent with our findings and the suggestion, albeit speculative at this stage, that skin adaptation to exercise or whole body heating may induce structural adaptations that are reflected as diminished maximal flux and CVC responses. Whilst changes in microvascular morphology in skeletal muscle may act to prolong transit time and enhance gas exchange, similar adaptation in the human skin would have the effect of enhancing heat exchange. Further studies should examine these hypotheses and the suggestion that exercise training modifies cutaneous microvascular morphology in humans.

Our finding that changes in CVC were only present in the limb that was exposed to repeated increases in blood flow, infers that shear stress may be an important stimulus for adaptation in human microvessels. Although shear stress decreases during passage down the arterial tree, it is nonetheless likely that the vessels imaged in the present study are exposed to physiologically relevant increases in levels of flow and shear in response to exercise. In vitro animal studies 
have reported that coronary arterioles between $80-130-\mu \mathrm{m}$ dilated by $50 \%$ and arterioles between $25-70 \mu \mathrm{m}$ dilated by $20-30 \%$ in response to shear stress $\{$ Kuo, $1995 \# 3198\}$. The vessels imaged in this study are likely $>30-50 \mu \mathrm{m}$ in diameter \{Fredriksson, 2009 \#3126;Braverman, 1989 \#3119\}. Also, Laughlin et al. reported that arterioles of smaller size possess more eNOS protein than those $>50 \mu \mathrm{m}$ \{Laughlin, $2003 \# 1560\}$. Regardless of the magnitude of shear the skin microcirculation was exposed to, blood flow through these areas was significantly increased, and this is associated with increased haemodynamic forces, that can act as mediators of adaptation \{Brown, $2003 \# 1635$ \}.

Limitations. Cuff inflation to $60 \mathrm{mmHg}$ was used to primarily attenuate blood flow in one limb during the exercise bouts. It is conceivable that cuff inflation may have induced unilateral venous distension which, in turn, elicited a reflex cardiovascular response impacting upon skin perfusion or flow \{Cui, 2012 \#3185\}. For example, previous studies which have inflated a cuff to 250mmHg and subsequently distended the veins via infusion, have reported a systemic reflex sympathetic impact \{Cui, 2011 \#3184;Cui, 2009 \#3186;Cui, 2012 \#3185\}. If such a reflex was induced in the present experiment, it might explain the contralateral uncuffed limb responses we observed. However, our experimental design was different to these studies because it involved cuff inflation to just $60 \mathrm{mmHg}$. Nonetheless, we performed a sub-study to determine potential systemic effects of unilateral cuff inflation to $60 \mathrm{mmHg}$ for 30 minutes. We observed no effects on blood pressure, HR or skin blood flows in the contralateral limb. We therefore think it unlikely, in this particular experiment, that the effects of cuff inflation could drive a large sympathetic response that would induce adaptation in contralateral skin blood flow responses, at rest, to local heating pre- versus post-training. Furthermore, any central reflex effect, if apparent, 
would manifest in both arms, cancelling out the likely impact in terms of our experimental design. If a unilateral vasoconstrictor reflex was evident in just the cuffed limb as a result of venous distension, then the microvascular adaptation to repeated episodic vasoconstriction should have been greater in the cuffed arm, yet the opposite was the case in this study. We observed no changes in skin microvascular responses to local heating at rest in the arm that had undergone cuffing during the training intervention (Figure 2 and 3). We therefore suggest that elicitation of reflex responses cannot explain the differences we observed between limbs. Another limitation is that we did not examine core temperature responses during acute cycle exercise, before and after exercise training. We cannot, therefore, relate our findings to changes in thermoregulatory capacity, but this was not our aim. The purpose of our study was related to intrinsic adaptations in cutaneous responses following exercise training, and the role of blood flow and temperature in such localised adaptations, which may be relevant to microvascular health and disease. A further limitation relates to our subject group selection. Whilst we studied subjects who were either sedentary or recreationally active (all $<5$ hrs regular exercise per week), we cannot exclude the possibility that more active or fit subjects may have exhibited different outcomes. Finally, the local heating protocol employed in the present study was not identical to that used by Black et al. that was shown to elicit a highly NO-dependent respense. Although Whilst the rate of temperature increase has been shown to have little effect on the absolute peak skin blood flow response $\{$ Choi, $2014 \# 3222\}$, the contribution of different vasodilator pathways to the response, such as NO and EDHF, may be different, therefore we cannot be certain that our results were largely $\mathrm{NO}$-dependent. Indeed, we cannot entirely rule out the possibility that the use of the use of a different local heating protocol_, particularly one that elicits an axon reflex response, may have induced distinct results to our heating protocolstudy. 


\section{Conclusion}

We found that cycle exercise training decreased forearm skin blood flow responses to localised heating. This adaptation appears to be dependent upon repeated increases in blood flow during exercise bouts, as adaptation in the skin microcirculation was not apparent in the contralateral limb in which flow responses and skin temperature were attenuated via cuff inflation. We speculate that an increase in cutaneous red cell transit time may result from structural adaptations that ${ }_{2}$ correspondingly ${ }_{2}$ lower maximal CVC responses. Although we cannot provide a definitive evidence-mechanism for such an adaptation-at this stage, due to methodological limitations with the direct assessment of cutaneous microvascular structure, future studies imaging technology () may be able to be required to-test this proposal_. 


\section{REFERENCES}




\section{Acknowledgements}

D.J.G was funded by the Australian Research Council (DP 130103793). D.H.J.T was a recipient

of the E. Dekker stipend (Netherlands Heart Foundation, 2009 T064). 
Figure Legends.

Figure 1. Heart rate (A, in beats per minute), and forearm skin temperature $\left(B\right.$, in $\left.{ }^{\circ} \mathrm{C}\right)$ and skin blood flow (C, in perfusion units (PU)) in the uncuffed (solid squares) and cuffed arm (open squares) before and during cycle exercise (at 2.5-min intervals) in healthy, young volunteers $(\mathrm{n}=6)$. Post-hoc significantly different at $\mathrm{P}<0.05$ from baseline $(*)$ or between the cuffed and uncuffed arm $\left(^{\#}\right)$. Error bars represent SE.

Figure 2. A representative response of skin blood flow change presented as cutaneous vascular conductance (CVC) during the gradual local heating protocol in the uncuffed (A) and cuffed (B) arms at Weeks 0 and 8 of the exercise intervention. PU: perfusion units; MAP: mean arterial pressure.

Figure 3. Forearm skin cutaneous vascular conductance $(\mathrm{CVC})$ at baseline $\left(33^{\circ} \mathrm{C}\right)$ and during gradual heating. Submaximal heating $\left(40^{\circ} \mathrm{C}\right), 42^{\circ} \mathrm{C}$ (Plateau) and $44^{\circ} \mathrm{C}$ (Max) are presented in the uncuffed (panel A) and cuffed arms (panel B) at 0 and following 8 weeks of exercise training (black and white bars, respectively) in healthy, young volunteers $(\mathrm{n}=14)$. Post-hoc significantly different at $\mathrm{P}<0.05$ from baseline $(*)$. Error bars represent SE. 